\title{
PSO-Based Design of RF Integrated Inductor
}

\author{
Pedro Pereira $^{1}$, Fernando V. Coito ${ }^{1}$, and Helena Fino ${ }^{2}$ \\ ${ }^{1}$ CTS, Uninova, Departamento de Engenharia Electrotécnica, Faculdade de Ciências \\ e Tecnologia, FCT, Universidade Nova de Lisboa, 2829-516 Caparica, Portugal \\ ${ }^{2}$ Departamento de Engenharia Electrotécnica, Faculdade de Ciências e Tecnologia, FCT, \\ Universidade Nova de Lisboa, 2829-516 Caparica, Portugal \\ pmrp@ieee.org, fjvc@fct.unl.pt, hfino@ieee.org
}

\begin{abstract}
This paper addresses an optimization-based approach for the design of RF integrated inductors. The methodology presented deals with the complexity of the design problem by formulating it as a multi-objective optimization. The multi-modal nature of the underlying functions combined with the need to be able to explore design trade-offs leads to the use of niching methods. This allows exploring not only the best trade-off solutions lying on the Pareto-optimum surface but also the quasi-optimum solutions that would be otherwise discarded. In this paper we take advantage of the niching properties of lbest PSO algorithm using ring topology to devise a simple optimizer able to find the local-optima. For the efficiency of the process analytical models are used for the passive/active devices. In spite the use of physics-based analytical expressions for the evaluation of the lumped elements, the variability of the process parameters is ignored in the optimization stage due to the significant computational burden it involves. Thus in the final stage both the Paretooptimum solutions and the quasi-optimum solutions are evaluated with respect to the sensitivity to process parameter variations.
\end{abstract}

Keywords: particle swarm optimization, multi-objective optimization, parameter variability, sensivity analysis, niching.

\section{Introduction}

The worldwide market on communications is still growing exponentially, mainly due to the ever scaling down of CMOS transistor sizes. This evolution yields the development of faster and cheaper circuits with increasing functionality. However it is widely recognised that as technology sizes continues to scale down, the variability in process parameters may cause significant deviations in device characteristics, forcing the adoption of new methodologies for the design of integrated circuits and systems. Generally, in nano-CMOS technology, imperfections in analog and digital circuits are a result of arbitrary errors in the implementation process. These errors, commonly referred as process's parameter variability, are consequence of several physical processes, that occur during fabrication, such as line edge roughness, random dopant fluctuations and oxide thickness variations [1]-[3]. Since process variability has strong influence on circuit reliability as well as in circuit lifetime, designers 
usually are tempted to use large design margins, degrading the circuit performance. In order to overcome these issues, reliable assessments must be done at design time [1]. In this paper, we propose using optimization-based design applied to an integrated planar inductor.

Stochastic optimization algorithms such as particle swarm optimization (PSO) algorithms have shown to be effective and robust optimization methods for solving difficult optimization problems [1]. The original and many existing forms of PSOs are designed for locating a single global solution. These algorithms typically converge to one final solution because of the global selection scheme used. However, the design problem under consideration is, by nature, "multimodal" meaning that there are multiple satisfactory solutions. For this type of optimization problems where multiple global and local optima exist, it is preferable to locate all global optima and/or some local optima that are also considered as being satisfactory. Techniques used to locate multiple optima (global or local) are referred to as "niching" methods. Niching PSO methods are able to promote and maintain multiple stable subpopulations within a single population, with an aim to locate multiple optimal or suboptimal solutions. Many of these niching methods require additional parameters, which are critical to the algorithm performance. Others have a high computational complexity, as they use global information calculated from the entire population [4]-[7]. The PSO algorithm used in the paper overcomes such difficulties by means of a local best (lbest) PSO algorithm with the population mapped onto a ring topology [2].

The integrated inductor design is formulated as a dual-objective optimization problem, where the parameter space is discretized by fixed step lengths which result from technical constraints. The lbestPSO algorithm is used to find a set of Paretooptimum and quasi-optimum solutions. The sensitivity of these solutions to parameter variation is analysed and a final set of solutions is selected to be submitted to the designer.

The remain of the paper is organized as follows. The novelty introduced by this paper, as well as the integrated planar inductor optimization strategy, is offered in Section 2. The lbestPSO algorithm is discussed in Section 3. Then, Section 4 is dedicated to the optimization examples. Finally, conclusions are offered in Section 5.

\section{Contribution to Value Creation}

The main objective of the optimization based tool presented is to generate the geometrical layout parameters of integrated spiral inductors. For this purpose two main concerns were considered. On one hand a technology-aware methodology is adopted where the discrete nature of the variables is accounted for as a way of restricting the search space to those points allowed by the technology. Further constraints arising from heuristic design rules are also considered. On the other hand the necessity for obtaining solutions in an efficient way led to the use of the inductor physical model, instead of using a simulation based approach. Several issues, such as process variability, technology-aware, accurate models, among others must to be taken into consideration in order to guarantee the accuracy of results. 
The novel contributions of this paper are as follows:

- It proposes an optimization-based design for nano-CMOS Planar Spiral Inductor, obtaining solutions in an efficient way based in analytical models, instead of using a simulation based approach;

- The integration of a multi-objective optimization procedure onto the design scheme to select a set of optimal and quasi-optimal trade-off solutions for the design. The integration from information on sensitivity to process parameter variations into the selection procedure is addressed.

\subsection{Planar Spiral Inductor Pi-Model}

The efficiency of the optimization tool is obtained through the use of an inductor model. For the sake of simplicity the pi-model, illustrated in Fig. 1.a., is adopted.

(a)

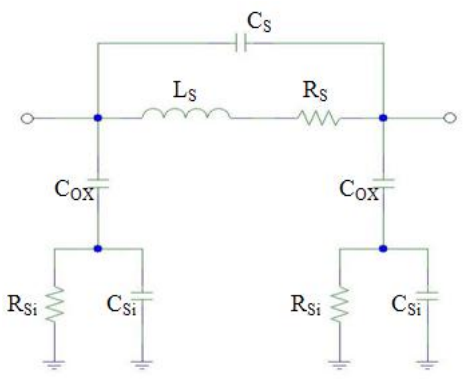

Fig. 1. a) Planar inductor pi-model; b) Layout of a square inductor

For the evaluation of the inductance, $L s$, several approaches have been proposed, based on a fitting process to values obtained experimentally [8], or through physicsbased equations [9]. In this tool the Modified Wheeler formula is used [8], where Ls is a function of the number of turns $(n)$, the internal diameter $\left(d_{i n}\right)$ and the track width ( $w$ ), as represented in Fig. 1.b. The value of $L s$ also depends on the inductor shape [8]. The evaluation of $\mathrm{R}_{\mathrm{s}}, \mathrm{R}_{\mathrm{si}} \mathrm{C}_{\mathrm{s}}, \mathrm{C}_{\mathrm{ox}}$, and $\mathrm{C}_{\mathrm{si}}$, is obtained with equations in [10] and [11].

The inductor quality factor $(\mathrm{Q})$ is usually adopted as the characteristic to be used when comparing inductors performance. For an accurate prediction of $\mathrm{Q}$, the parasitic effects cannot be neglect. The pi-model presented in Fig. 1 takes into account a set of various parasitic and loss elements, allowing to write $(Q)$ as function of the passive components. The $\mathrm{Q}$ is usually calculated reducing the two ports of the pi-model, to a single port by grounding the second port (shadow branch) [13]. This configuration aims to simplify the analysis of the $\mathrm{Q}$ behaviour. The $\mathrm{Q}$ is analytical obtained by

$$
Q=\frac{\omega L_{S}}{R_{S}} \frac{R_{P}}{R_{P}+\left[\left(\frac{\omega L_{S}}{R_{S}}\right)^{2}+1\right] R_{S}}\left[1-\frac{R_{S}^{2}}{L_{S}}\left(C_{S}+C_{P}\right)-\omega^{2} L_{S}\left(C_{S}+C_{P}\right)\right],
$$

where 


$$
\begin{aligned}
& R_{P}=\frac{1}{\omega^{2} C_{o x}^{2} R_{s i}}+\frac{R_{s i}\left(C_{o x}+C_{s i}\right)^{2}}{C_{o x}^{2}} \\
& C_{P}=C_{o x} \frac{1+\omega^{2}\left(C_{o x}+C_{s i}\right) C_{s i} R_{s i}^{2}}{1+\omega^{2}\left(C_{o x}+C_{s i}\right)^{2} R_{s i}^{2}} .
\end{aligned}
$$

\subsection{Particle Swarm Optimization Applied to Integrated Inductor Design}

PSO is a swarm intelligence technique originally developed from studies of social behaviors of animals or insects [13]. Since its inception PSO has gained increasing popularity among as a robust and efficient technique for solving optimization problems.

PSO is based on the evolution of a population of particles. It manipulates each particle position (i.e., a candidate solution) within the search space based on its velocity, some previous best positions it has found so far, and previous best positions found by its neighbouring particles. In a canonical PSO, the velocity of each particle is modified iteratively by its personal best position (i.e., the position giving the best fitness value so far), and the position of best particle from the entire swarm. As the algorithm evolves the particles are attracted towards a single global solution.

In problems like the inductor design, however, multiple satisfactory solutions exist for which the selection criterion is difficult to formalize into a performance index. Thus, it is important to have a tool able to pick the set of satisfactory solutions instead of only a global one. Here we take advantage of the niching properties of a lbestPSO algorithm with a ring topology population map [14] to find the set of solutions. We seek to simultaneously maximize the inductors quality factor $(Q)$ while minimizing its deviation from the specified value $\left(\left|L_{s}-L_{\text {specified }}\right| / L_{\text {specified }}\right)$.

The parameter search space consists of the number of turns $(n)$ of the inductor, the internal diameter $\left(d_{i n}\right)$, the track width $(w)$ and the inductor shape. Technical constraints limit the precision for each of these parameters. This leads to the discretization of the search space. The sensitivity of these solutions to parameter variation is analysed and a final set of solutions is selected to be submitted to the designer.

\section{Particle Swarm Optimization Algorithm}

In PSO algorithms a set of particles is set to drift along the search space. At iteration $k$ the displacement of the $i^{\text {th }}$ particle within the search space is characterized by its position $\left(\boldsymbol{x}_{k}^{i}\right)$ and velocity $\left(\boldsymbol{v}_{k}^{i}\right)$. Each particle position is evaluated according to the performance index to optimize. The particle velocity is updated from a comparison between its current position, its best past position and the best past position from its group:

$$
\boldsymbol{v}_{\boldsymbol{k}+\mathbf{1}}^{\boldsymbol{i}}=\alpha \boldsymbol{v}_{\boldsymbol{k}}^{\boldsymbol{i}}+c_{1} \operatorname{rand}()\left(\boldsymbol{p}_{\boldsymbol{k}}^{\boldsymbol{i}}-\boldsymbol{x}_{\boldsymbol{k}}^{\boldsymbol{i}}\right)+c_{2} \operatorname{rand}()\left(\boldsymbol{p}_{\boldsymbol{k}}^{\boldsymbol{g}}-\boldsymbol{x}_{\boldsymbol{k}}^{\boldsymbol{i}}\right)
$$




$$
x_{k+1}^{i}=x_{k}^{i}+v_{k+1}^{i}
$$

where $w, c_{1}$ and $c_{2}$ are real valued parameters; $\boldsymbol{p}_{\boldsymbol{k}}^{\boldsymbol{i}}$ is the location corresponding to the best position on particle $i$ history; $\boldsymbol{p}_{\boldsymbol{k}}^{\boldsymbol{g}}$ is the location corresponding to the best position ever found by any particle in the group to which particle $i$ belongs; rand() is a real valued random vector, uniformly distributed in the range [0,1]. In the canonical algorithm $\boldsymbol{p}_{\boldsymbol{k}}^{g}$ is evaluated from the entire population.

\subsection{Niching PSO Using Ring Topology}

Let the swarm of particles be mapped on to a ring topology as presented in Fig. 2 . Associate with each particle not only a personal best $\boldsymbol{p}_{\boldsymbol{k}}^{\boldsymbol{i}}$ but also a group best $\boldsymbol{p}_{\boldsymbol{k}}^{\boldsymbol{g}, \boldsymbol{i}}$. The evaluation of each particle group best $\left(\boldsymbol{p}_{\boldsymbol{k}}^{g, \boldsymbol{i}}\right)$ only takes into account its neighbouring particles (for example, to update $\boldsymbol{p}_{\boldsymbol{k}}^{g, \mathbf{3}}$ the personal best of 3 particles are considered: $\boldsymbol{p}_{\boldsymbol{k}}^{\mathbf{2}}, \boldsymbol{p}_{\boldsymbol{k}}^{\mathbf{3}}$ and $\left.p_{k}^{4}\right)$.

The fact that each particle interacts only with its two neighbours slows down the communication within the swarm. As shown in [11] this leads the particles to associate in clusters around the local optima. Slow communication is a desirable feature on an algorithm which aims to locate multiple optima as it allows the particles to thoroughly search in its local neighbourhood.

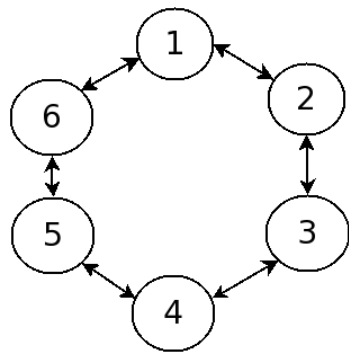

Fig. 2. PSO swarm ring topology with 6 particles

When the algorithm stops the set of group best positions $\left\{\boldsymbol{p}_{\boldsymbol{k}}^{g, i}: i=1, \cdots, n\right\}$ corresponds to the set of local optimal solutions.

\subsection{PSO and Discrete Search Space}

Technical constraints limit the feasible values for the design parameters, leading to a discrete search space. As expressions (4) and (5) do not account for this discrete nature of the parameters, the updating of the particle position needs to be modified.

The modification amounts to compare the particle position determined from expressions (4) and (5) and replacing it by the nearest discretized value. Once the actual position is determined the particle performance is evaluated. This insures that the algorithm optima truly correspond to feasible solutions. 


\section{Optimization of Inductor Design}

In this section we consider the design of a $5 \mathrm{nH}$ inductor. The design concerns the evaluation of four independent parameters, namely the track width $(w)$, the number of turns $(n)$, inductor shape $\left(N_{\text {side }}\right)$, and the internal diameter $\left(d_{i n}\right)$. Design constrains that lead to the discretization of the parameter space are presented in Table 1 . The design is also subjected to other technical constrains as described in [15].

\subsection{Dual-Objective Optimization}

The optimization scheme has two simultaneous objectives

- $\quad$ To maximize the inductors quality factor $(Q)$.

- To minimize the inductance deviation $\left(\left|L_{s}-L_{\text {specified }}\right| / L_{\text {specified }}\right)$.

These objectives may not be simultaneously fulfilled as improving the inductors precision generally leads to a decrease on the quality factor. Therefore some trade-off needs to be found.

To this purpose the niching PSO algorithm from section 3 is applied to 2 different swarms. One is aimed to minimize the inductance deviation. The other one aims to maximize $Q$, with the additional constrain that the inductance deviation must not become greater than $5 \%$.

The local optimal solutions obtained from both swarms is merged into Fig. 3.a. Only solutions corresponding to a precision under $1 \%$ are presented. It must be stressed that, as the parameter space is of discrete nature, only a discrete set of solutions is feasible.

Analysing the results it is easily found that only 5 of the displayed solutions correspond to an optimal trade-off (marked as O). For all the other solutions it is possible to increase the quality factor without losing precision (or vice-versa). From a Pareto-optimal approach these 5 solutions correspond to the set from which the designer ought to select.

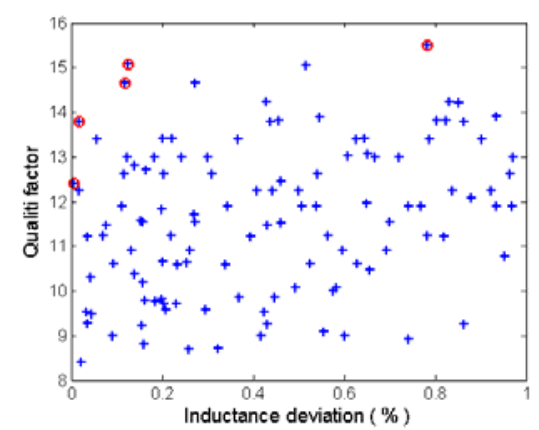

(a)

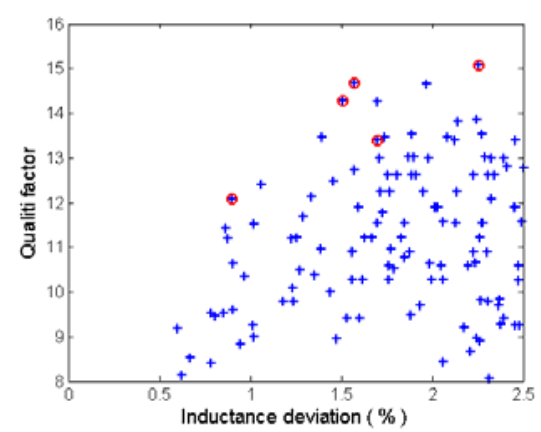

(b)

Fig. 3. a) Local optima from niching PSO; b) Post-processing of the local optima (worst case approach). The $\mathbf{O}$ corresponds to the Pareto-optima solutions 
Table 1. Spiral Inductor Design Constraints

\begin{tabular}{cccc}
\hline Parameter & Min & Step & Max \\
\hline$w(\mu \mathrm{m})$ & 5.0 & 0.5 & 100.0 \\
$d_{\text {in }}(\mu \mathrm{m})$ & 20.0 & 0.5 & 200.0 \\
$n$ & 1.5 & 1.0 & 15.5 \\
$N_{\text {side }}$ & Square (4) / Hexagonal (6) / Octagonal (8) \\
\hline
\end{tabular}

\subsection{Analysis of the Sensitivity to Parameter Variation}

In this section we present a simple study to illustrate the fact that the set of solutions to take under consideration should not be restricted to the Pareto-optimal set from Fig. 3.a.

We will assume that the actual implemented values for the track width $(w)$ and the internal diameter $\left(d_{i n}\right)$ may differ from the design values. The maximum tolerance for each of these parameters is assumed to be equal to the value of step from Table 1.

For each of the local solutions presented in Fig. 3.a the values for Q and precision are re-evaluated taking into account these tolerances. The results from this procedure are determined through a worst case approach (Fig. 3.b).

Not surprisingly the worst case inductance deviation values are larger than in the error free case. More relevant, however, is the fact that not all the previous Paretooptimal solutions still appear at the new set of best trade-off solutions (Table 2). Also, from Fig. 3.b, it is clear that this worst case procedure leads to the inclusion of new solutions as possible design choices.

Of course the proposed procedure may be integrated in the optimization algorithm, however, its inclusion would heavily increase the overall computational burden. It is therefore preferable to use it to post-process the optimization solutions.

Table 2. Spiral Inductor Optimization Results

\begin{tabular}{cccccccc}
\hline$Q$ & $Q_{\text {worst case }}$ & $\begin{array}{c}\text { Inductor } \\
\text { deviation } \\
(\%)\end{array}$ & $\begin{array}{c}\text { Inductor } \\
\text { deviation } \\
\text { (worst case) }\end{array}$ & $w(\mu \mathrm{m})$ & $d_{\text {in }}(\mu \mathrm{m})$ & $n$ & $N_{\text {side }}$ \\
\hline 12.3978 & 12.0862 & 0.0048 & 0.8978 & 8.7500 & 76.7500 & 5.5000 & 6.0000 \\
13.7986 & 13.3919 & 0.0169 & 1.6995 & 9.0000 & 46.7500 & 6.5000 & 8.0000 \\
14.6640 & 14.2776 & 0.1187 & 1.5074 & 10.5000 & 56.2500 & 5.5000 & 4.0000 \\
15.0764 & 14.6753 & 0.1254 & 1.5703 & 10.7500 & 55.7500 & 5.5000 & 4.0000 \\
15.4917 & 15.0764 & 0.7821 & 2.2540 & 11.0000 & 55.5000 & 5.5000 & 4.0000 \\
\hline
\end{tabular}

\section{Conclusions}

The work addressed the design of RF integrated inductors as an optimization-based problem. The methodology presented deals with the complexity of the design by formulating it as a multi-objective optimization problem. 
The fact that different combinations of the design parameters may lead to similar results enhances the need for methods capable of dealing with multiple local optimal solutions. The local-optima were determined using a niching PSO algorithm with ring swarm topology. The PSO algorithm was used to simultaneously optimize the precision and quality factor of the inductor, while taking into account the discrete nature of the design parameters.

A simple example showed that the outcome of the optimization algorithm must include both the Pareto-optimal solutions and the set of quasi-optimal solutions.

\section{References}

1. Maricau, E., Gielen, G.: Computer-Aided Analog Circuit Design for Reliability in Nanometer CMOS. IEEE Journal on Emerging and Selected Topics in Circuits and Systems 1, 50-58 (2011)

2. Ghai, D., Mohanty, S., Kougianos, E.: Design of Parasitic and Process-Variation Aware Nano-CMOS RF Circuits: A VCO Case Study. IEEE Transactions on Very Large Scale Integration (VLSI) Systems 17, 1339-1342 (2009)

3. Ghai, D., Mohanty, S., Kougianos, E.: Parasitic Aware Process Variation Tolerant Voltage Controlled Oscillator (VCO) Design. In: 9th International Symposium on Quality Electronic Design (ISQED), pp. 330-333 (2008)

4. De Jong, K.A.: An analysis of the behavior of a class of genetic adaptive systems. Ph.D. dissertation, Univ. Michigan, Ann Arbor, MI (1975)

5. Goldberg, D.E., Richardson, J.: Genetic algorithms with sharing for multimodal function optimization. In: Proc. 2nd Int. Conf. Genet. Algorith., Cambridge, MA, pp. 41-49 (1987)

6. Harik, G.R.: Finding multimodal solutions using restricted tournament selection. In: Proc. 6th Int. Conf. Genet. Algorith., pp. 24-31. Morgan Kaufmann, San Francisco (1995), http://citeseer.ist.psu.edu/harik95finding.html

7. Streichert, F., Stein, G., Ulmer, H., Zell, A.: A Clustering Based Niching EA for Multimodal Search Spaces. In: Liardet, P., Collet, P., Fonlupt, C., Lutton, E., Schoenauer, M. (eds.) EA 2003. LNCS, vol. 2936, pp. 293-304. Springer, Heidelberg (2004) ISBN: 978-3-540-21523-3

8. Mohan, S., Hershenson, M., Boyd, S., Lee, T.: Simple Accurate Expressions for Planar Spiral Inductances. IEEE Journal of Solid-State Circuits 34 (1999)

9. Jenei, S., Nauwelaers, B., Decoutere, S.: Physics-Based Closed-Form Inductance Expression for Compact Modelling of Integrated Spiral Inductors. IEEE J. Solid-State Circuits 37, 77-80 (2002)

10. Choi, Y., Yoon, J.: Experimental Analysis of the Effect of Metal Thickness on the Quality Factor in Integrated Spiral Inductors for RF ICs. IEEE Electron Device Lett. 25, 76-79 (2004)

11. Murphy, O.: Advanced Physical Modelling of Multilayer Inductors for CMOS RF FrontEnd Applications. PhD Thesis, Univ. College Cork - National University of Ireland (2005)

12. Pereira, P., Fino, M.H., Coito, F., Ventim-Neves, M.: GADISI - Genetic Algorithms Applied to the Automatic Design of Integrated Spiral Inductors. In: Camarinha-Matos, L.M., Pereira, P., Ribeiro, L. (eds.) DoCEIS 2010. IFIP AICT, vol. 314, pp. 515-522. Springer, Heidelberg (2010)

13. Kennedy, J., Eberhart, R.: Swarm Intelligence. Morgan Kaufmann, San Mateo (2001)

14. Li, X.: Niching Without Niching Parameters: Particle Swarm Optimization Using a Ring Topology. IEEE Trans. Evolutionary Computation 14(1), 150-169 (2010)

15. Pereira, P., Helena Fino, M., Coito, F., Ventim-Neves, M.: RF integrated inductor modeling and its application to optimization-based design. J. Analog Integrated Circuits and Signal Processing (2011) 